\title{
Editorial: Advancements in Pediatric Hepatology - Approach Based Diagnosis and Management
}

\author{
Girish Gupte $^{1}$ - Anshu Srivastava ${ }^{2}$
}

Received: 3 August 2016/Accepted: 3 August 2016/Published online: 4 October 2016

(C) Dr. K C Chaudhuri Foundation 2016

It is our great pleasure and privilege to bring together this special issue on pediatric gastroenterology and hepatology. Pediatric gastroenterology and hepatology fields, as a pediatric sub-specialty, has developed and made significant progress in the last decade within India. Pediatricians in teaching hospitals, non-teaching hospitals and private clinics are now faced with children presenting with symptoms related to complex liver conditions. Investigations and management of these conditions can be challenging and expensive and hence understanding of the common conditions and tailoring the investigations is of vital importance in management of the conditions.

This special issue tries to address a practical approach to the problems within pediatric hepatology.

The article on 'Space occupying lesions in the liver' is a comprehensive and detailed documentation of the various causes of this uncommon condition [1]. Although the conditions are rare, arriving at a diagnosis can be a challenge. There is a wide variety of imaging modalities available and the article tends to provide an insight into the radiological investigation to request and interpretation of the changes seen on imaging. The correct radiological investigation can have a huge impact on the correct timing to commence treatment which can in effect influence and improve the outcome for the patients.

Girish Gupte

girish.gupte@bch.nhs.uk

1 Liver Unit (Including Small Bowel Transplantation), Department of Gastroenterology and Nutrition, Birmingham Children's Hospital, Steelhouse Lane, Birmingham B46NH, UK

2 Department of Pediatric Gastroenterology, Sanjay Gandhi Postgraduate Institute of Medical Sciences, Raebareli Road, Lucknow, Uttar Pradesh, India
The article on 'Hepatitis B and Hepatitis C in children' provides a simplistic approach to the understanding of these subtle diseases which have a huge influence on the long term outcome as an adult [2]. There is a clear and succinct explanation for the interpretation of the serological tests which in turn influence appropriate management. The article provides answers to common questions and clinical scenarios encountered by the non-specialist pediatricians in a private clinic setting.

The article on "Jaundice and pruritus" details the reasons for jaundice and the investigations needed in different clinical scenarios [3]. It presents the scientific basis for using medications in the management of pruritus which is one of the most difficult symptoms to treat as there is no direct medical cure. The article also draws the attention of the readers to the uncommon conditions presenting with jaundice.

Metabolic liver disease is always considered to be a 'minefield' area which is difficult to navigate through the vista of presenting symptoms children can present with and arrange the appropriate investigations [4]. The article is full of practical advice for the practicing pediatrician with 'red flag signs' and different problems that children can present with and rationale of limiting the investigations to reach a diagnosis. The article also has different clinical scenarios (protocols) that children can present with and demonstrates the key investigations that need to be done to reach a diagnosis. The article mentions about the current advances in management.

The article on 'Ascites in children' elaborates on the key causes of ascites and the investigations and management of uncomplicated and complicated ascites [5]. The article helps to understand biochemical interpretation of ascitic fluid which can be of overwhelming importance towards deciding about further investigations and establishing the diagnosis. The article updates on the treatment options for ascites. 
We are grateful to the national and international authors for their own personal time and effort towards the contribution to the issue making it reader friendly. We have enjoyed co-coordinating the issue despite the challenges and hope that the readers appreciate the succinct, practical approach which can be adapted to individual needs.

Conflict of Interest None.

Source of Funding None.

\section{References}

1. Thyagarajan MS, Sharif K. Space occupying lesions in the liver. Indian J Pediatr. 2016. doi:10.1007/s12098-016-2240-x.

2. Malik R, Hardikar W. Hepatitis B and C in children. Indian J Pediatr. 2016. doi:10.1007/s12098-016-2076-4.

3. Jagadisan B, Srivastava A. Child with jaundice and pruritus: how to evaluate? Indian J Pediatr. 2016. doi:10.1007/s12098-016-2058-6.

4. Alam S, Sood V. Metabolic liver disease: when to suspect and how to diagnose? Indian J Pediatr. 2016. doi:10.1007/s12098-016-2097-z.

5. Bavdekar A, Thakur N. Ascites in children. Indian J Pediatr. 2016. doi:10.1007/s12098-016-2168-1. 